\title{
A priori ohne Apriori A-moderne, Sprachpragmatik und Geographie
}

\section{Wolfgang Zierhofer, Nijmegen}

\section{Punkt, Linie, Kreis, Netz, wir}

Wir arbeiten an unserem Übergang vom Kreis zum Netz.

Wir sind kein majestätischer Plural, keine Kaschierung des selbstbezüglichen Ichs und schon gar kein durch Satzungen verfasstes oder durch Gesinnung verschweißtes Kollektiv, sondern «links» im Internet der Existenz. Wir wollen uns von der Seite des Netzes aus verstehen. In der Welt der Punkte, Linien und Kreise wurden wir fein säuberlich in verschiedene Zeiten, Orte, Körper, Kulturen, Geschlechter, Ansätze, Sprachen, Theorien, Personen aufgeteilt. Indem wir unserem Aufteilen Aufmerksamkeit schenken, gewinnen wir unsere netzwerkartige Identität zurück, ohne die durch das Teilen produzierten Ordnungen negieren zu müssen.

Wir können es uns erlauben, zu versuchen, zugleich die Dinge auf den Punkt zu bringen, der Linearität der Sprache zu folgen, um die Zirkularität herum zu schreiben und uns als Eigenheiten abzugrenzen, indem wir Beziehungen aufbauen. Wir sind nicht mehr gezwungen, etwas zu setzen oder von einem Gesetzten auszugehen, und schon gar nicht, daran festzuhalten. Wir können uns damit begnügen, in Rechnung zu stellen, dass unser Tun seine Konsequenzen hat.

«A priori ohne Apriori» - das kann freilich als Paradox gelesen werden. Dann wäre es ein Kreis. Fehlt nicht auch ein Ausrufezeichen? Es wäre also eine Linie, der $\mathrm{zu}$ folgen ist. Ist es nicht selbst eine Setzung? Somit wäre ein Ausgangspunkt bestimmt worden. Diesen Auslegungen können wir uns nicht verschließen. Mit ihrer Möglichkeit müssen wir leben. Aber angenommen, wir würden uns weigern, «a priori ohne Apriori» ausschließlich als Paradox, Norm oder Apriori zu lesen, was wäre es dann?

\section{Die Moderne empfängt Post - woher?}

Postmoderne, Postmetaphysik, Poststrukturalismus, Postindustrialismus, Posthistorie, Postmaterialismus an Versuchen, sich von der Moderne oder von einigen ihrer Besonderheiten abzusetzen, fehlt es wahrlich nicht. Sobald das Präfix «post» im Sinne einer Über- windung verstanden wird, schleicht sich allerdings die Moderne als sich selbst konstituierende Epoche, als selbstgewisser Fortschrittszusammenhang, schon wieder durch die Hintertüre ein. Deshalb spricht Wolfgang WelsCH (1986) auch von der postmodernen Moderne. Das «post» zeitlich zu deuten, wäre problematisch, denn damit bliebe man implizit jenen typisch modernen Denkmustern verhaftet, die man durch das «post» explizit verabschieden will.

Konsistenter erscheint die Interpretation postmodernen Denkens als «dezentrierte» Perspektive innerhalb der Moderne. Postmoderne wäre folglich ein Versuch, zu den eigenen Grundlagen auf Distanz zu gehen, sie der Selbstverständlichkeit zu entziehen und sie der Kritik (mit zunächst modernen Mitteln freilich) zuzuführen. Die Postmoderne ließe sich dann als zunächst ahistorisch verstandener Denk- und Argumentationszusammenhang, als Idealtypus sozusagen, verstehen.

Andere Begriffe, die durchaus mit der Postmoderne in engem Zusammenhang stehen, legen hingegen zumindest eine teilweise zeitliche Interpretation nahe: «Poststrukturalismus» beispielsweise, bezieht sich einerseits auf einen «Generationenwechsel» in der französischen Philosophie, der aber zugleich eine Dezentrierung modernen Denkens, also eine Postmoderne, darstellt. Denn die Suche nach reinen, universellen Strukturen des Denkens, Sprechens, Zusammenlebens verkörpert einen Kernbestand klassisch modernen Denkens, dessen Ansprüche an Gültigkeit und Reichweite im Rahmen der Postmoderne-Debatte zurückgewiesen werden. Auch ein Begriff wie Postindustrialismus bezieht sich auf eine historisch bestimmbare Verschiebung der «Gewichte» von Wirtschaftssektoren. Doch ist damit kein Ausstieg aus der industriellen Produktion verbunden - ebensowenig, wie zuvor die Industrialisierung die Landwirtschaft obsolet werden ließ.

Das «post» bezieht sich also häufig auf Entwicklungen, die nicht als ein "Nachher», sondern als ein «Sowohl-als-auch» zu verstehen sind. Wie sollen nun aber Autoren verstanden werden, die für sich das Label «a-modern» reklamieren? Haben Sie einen Weg gefunden, vollständig aus der Moderne auszusteigen? Sind sie durch und durch nicht-mehr-modern?

Die Bezeichnung a-modern bezieht sich auf Bruno LATOURs Buch «Wir sind nie modern gewesen» (1995). Dieses Buch systematisiert Entwicklungen in Wissen- 
schaftssoziologie und Wissenschaftstheorie, die auch unter den Bezeichnungen «science studies» und «actor network theory» bekannt geworden sind (vgl. Law \& Hassard 1999, Haraway 1995). Angesichts dieses Buchtitels könnte man nun versucht sein, von Prämoderne zu sprechen, doch auch diese Begriffswahl würde die Zeitkonzeption der Moderne, d.h. ihr Selbstverständnis als vorläufiger Endzustand einer glorreichen Entwicklung, implizieren. Genau das ist nicht gemeint, und deshalb hätte LATOUR vielleicht auch einen Titel im Präsens formulieren sollen:Wir sind nicht so modern, wie wir meinen. Selbstverständlich kommt es den A-modernen nicht in den Sinn, ihre geistigen Ahnen zu leugnen. Vielmehr fühlen sie sich ihren historischen Wurzeln dermaßen verbunden, dass sie die Möglichkeit einer eindeutigen Historisierung in Frage stellen. Das Gestern, Heute und Morgen durchdringt uns, und erst die Art dieser Durchdringung macht es uns möglich, das Gestern, Heute und Morgen auf unsere je spezifische Weise zu denken.

Analoges gilt für jeden anderen Begriff, insbesondere für die Unterscheidungen von Kultur und Natur, von Objekt und Subjekt, von Individuum und Gesellschaft, von Mann und Frau und all die anderen Formen, mit denen sich das moderne Denken seiner Identität zu vergewissern versucht(e). Doch genau dieses «Durchdringen» der «Dinge», diese Hybridität aller Identitäten, das wird vom modernen Denken in Abrede gestellt. Indem es die Existenz hybrider «Wesen» bestreitet, kann sich das moderne Denken von seinen Vorformen absetzen und die Moderne als Epoche deklarieren. Und weil das Denken in isolierten Einheiten für die Modernen zuvor unbekannte Möglichkeiten eröffnet, diese Einheiten auf andere Weise zu kombinieren, weil dies die entscheidende mentale Grundlage der technologischen Revo- lution und der emanzipatorischen Umgestaltung sozialer Verhältnisse ist, kann sich die Moderne auch selbst als Fortschrittszusammenhang begreifen.

Nicht die Möglichkeit, die Dinge so zu sehen, wird von den A-modernen bestritten, sondern nur der moderne Anspruch, es ausschließlich so sehen zu können. Es ist also eigentlich das moderne Denken, das sich dezidiert von jeglicher Form nicht-modernen Denkens absetzt, weil es sich selbst durch diese Unterscheidung als modernes Denken konstituiert. Vor diesem Hintergrund wird auch eher verständlich, weshalb die «post»-Rhetorik laufend zeitlich interpretiert wird: Sie wird von einer partiell modernen Warte aus rezipiert und als Ende der eigenen Epoche verstanden. Für die A-modernen trägt die Figur der Epoche nicht sehr weit: Sie begreifen die Moderne nicht als zeit-räumlich abgrenzbare Kultur, und daher können sie sich auch nur sehr bedingt zeitlich von der Moderne absetzen. Sie sehen sich damit allerdings in scharfem Kontrast zu den Absetzungsversuchen der Moderne selbst. So gesehen, kann die Moderne nur als Abstraktion in Reinkultur bestehen. Nur insofern als ein Idealtypus «Moderne» zur Leitfigur der normativen Selbstbeschreibung während einer gewissen Zeit - ungefähr seit Ende des 18. Jahrhunderts - und im Rahmen eines räumlich mehr oder weniger abgrenzbaren Kommunikationszusammenhanges - im Okzident - dominant werden konnte, kann von Moderne als Epoche gesprochen werden. Auf diese Weise wird mitgedacht, was der Idealtypus auszuklammern versucht.

Dem Konstitutionsmodus der Modernen, der für die Organisation des Lebens, insbesondere für die Verfügbarmachung der Natur und für die Autonomie des Subjektes, so entscheidend ist, wird von den A-modernen der Status als Verfassung abgesprochen.
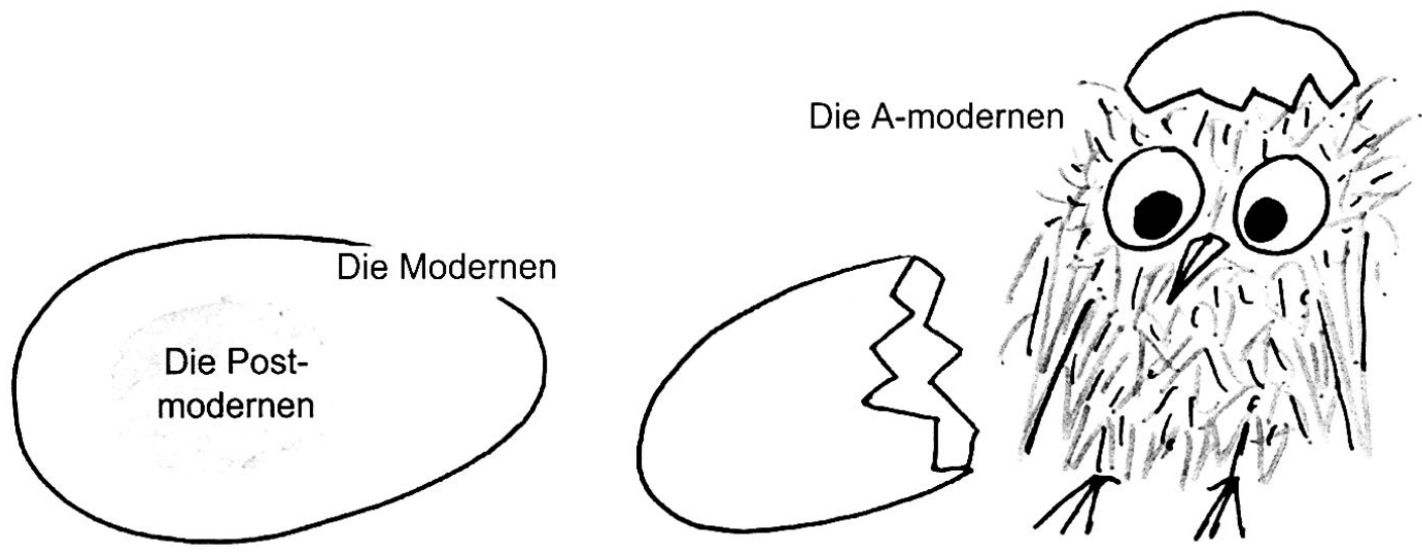

Abb. 1: Modernes Ei und a-modernes Küken

Modern egg and a-modern chick

Oeuf moderne et poussin non-moderne 
Sie haben zwar nicht mit dem modernen Verständnis von Welt und Erkenntnis gebrochen, bestreiten jedoch seinen Führungsanspruch: Es ist eine mögliche Perspektive mit begrenzter Reichweite. Mit den Modernen und Postmodernen teilen die A-modernen jedoch ein aufklärerisches, kritisch-emanzipatorisches Anliegen. Je a-moderner Moderne und Postmoderne denken, desto schwieriger fällt es ihnen, eindeutige Grenzen zwischen Moderne, Postmoderne und A-moderne zu ziehen.

Wenn wir mit Liebe zu Metaphern die Moderne einmal als Ei betrachten wollen, dann sind die Vertreter des modernen Denkens das Weiße des Eies. Sie sind notwendig, einigermaßen nahrhaft, aber geschmacklos. Insofern sich die Postmodernen von den Modernen absetzen, stellen sie das Gelbe vom Ei dar. Mit den Modernen verbindet sie allerdings die Konstitution als Ei: Aus ihrer Sicht kann es nämlich nur weiß oder gelb, oder allenfalls noch Rührei geben. Alles andere liegt außerhalb der Schale und übersteigt ihre Vorstellungskraft. Das Ei kann sich keine Vorstellung vom Küken machen. Das Küken hingegen, frisch dem Ei entschlüpft und zwischen den Bruchstücken der Schale sitzend, kann sich sehr wohl als Durchdringung von weiß und gelb, als hybrides Wesen begreifen. Es würde dem Küken auch nie in den Sinn kommen, die Existenz des Eies leugnen zu wollen. Und dennoch kann es sich selbst nicht einfach als Ei betrachten. Sind wir bereit, die A-modernen als Küken zu verstehen?

\section{Die a-moderne Konstitution der Realität}

Im Zentrum des a-modernen Weltverständnisses steht eine zutiefst pragmatische und konstruktivistische Konzeption: Die Fähigkeit, irgendwelche «Dinge» oder Entitäten in der Welt unterscheiden zu können, wird nicht den Sachverhalten zugerechnet, die den erkennenden Instanzen vorausliegen, sondern vollumfänglich den Praktiken der Erkennenden selbst. In diese Praktiken sind allerdings die unterschiedlichsten Sachverhalte involviert. Jede Differenzierungsfähigkeit wird daher als Ergebnis einer Geschichte von Erkenntnisanstrengungen betrachtet, aber nicht als Struktur einer Welt, die der Erkenntnis vorgegeben wäre. Über die Welt "an sich» werden keine Aussagen gemacht (also auch nicht die, dass sie nicht direkt zugänglich sei). Mit anderen Worten: Die A-modernen operieren ohne transzendentale Apriori.

Selbstverständlich muss jede Rede, auch diejenige der A-modernen, eine Grundlage voraussetzen: Ohne Semantik, Grammatik, Syntax, Logik, Annahmen über die Welt und die Fähigkeiten potenzieller Kommunikationsteilnehmer kommt keine Diskussion zustande. Entscheidend ist, dass diesen Voraussetzungen kein transzendentaler Status beigemessen wird: Sie gelten zunächst als kontingente Differenzierungen. Das bedeutet aber auch, dass umgekehrt, die Möglichkeit transzendentaler Apriori nicht ausgeschlossen werden kann, denn diese Prämisse würde sogleich selbst transzendentalen Status beanspruchen. Das scheinbare Paradox «a priori ohne Apriori» löst sich also auf, wenn der empirische/transzendentale Status von Apriori miteinbezogen wird.

Ist das nicht eine typisch moderne Sichtweise? Ja und nein, denn sie entspricht zwar dem Anspruch der Modernen, aber nicht ihren Praktiken. Die Modernen geben vor, alles zu hinterfragen, alle sozialen Verhältnisse zu verflüssigen und Diskriminierungen entgegenzutreten (HABERMAS 1990, BECK 1991). Zugleich reproduzieren sie in ihren, für die Grundordnung ihres Daseins konstitutiven Reden, eine Art Kastensystem, das auf transzendentalen Apriori beruht: Mit dem «doppelten» Code von Kultur/Natur und höherwertig/minderwertig operierend, wird die Welt als Erfahrungszusammenhang und das Zusammenleben in dieser Welt durch parallel ansetzende Assoziationen (wie: Logos/Emotion, Geist/Körper, Mann/Frau, Zentrum/Peripherie, Selbst/Andere usw.) geordnet, wobei jeweils die eine Seite der Ordnung inferior gesetzt wird (vgl. Zierhofer 1999: 6f.). Da dieses System von Ausschlüssen und Unterordnungen dem emanzipatorischen Anspruch der Modernen nicht standhält - darin liegt übrigens das argumentative Kapital vieler sozialer Bewegungen - können wir mit LATOUR davon ausgehen, wir seien nie wirklich modern gewesen. Die A-modernen begreifen sich also als konsequente Weiterführung, und daher Radikalisierung, der konstruktivistischen Moderne.

Gemäß Bruno Latour operieren die Modernen mit einer kategorialen und transzendentalen Unterscheidung von Natur und Kultur (vgl. Abb. 2). Kategorial ist diese Unterscheidung, weil es nichts gibt, was zugleich beiden Mengen oder Klassen angehören könnte. Außer Gott, der nicht von dieser Welt ist, weil er ihr transzendental vorausliegt, gibt es jenseits von Natur und Kultur kein Drittes. Transzendental ist diese Dichotomie, weil sie nicht selbst als Produkt menschlicher Erkenntnispraktiken, nicht als Resultat eines Kontextes des Handelns oder Sprechens, nicht als verhandelbare Größe begriffen wird.

Natur und Kultur auf diese Weise unterscheiden zu können, ist die große Erfindung der Moderne. Sie ermöglicht es, in der Welt scharf zwischen den unveränderlichen Gesetzen der Natur und den kontingenten Setzungen der Menschen zu unterscheiden. Somit lassen sich die Gesetze der Natur für die unterschiedlichsten gesellschaftlichen Zwecke nutzbar machen. Andererseits lassen sich Herrschaftsstruktu- 
ren nicht länger durch Verweis auf göttliche Fügungen legitimieren, sondern müssen als kontingente Praktiken durch Zustimmung legitimiert (oder gewaltsam durchgesetzt) werden. Beide Formen der Emanzipation, die technologische wie die demokratische, beruhen in wesentlichen Zügen auf der transzendentalen Dichotomie von Natur und Kultur.

Den Vormodernen ist es nicht in den Sinn gekommen, Natur und Kultur auf diese Weise zu unterscheiden. Sie sind nicht fähig, im selben Sinn Technologien und soziale Institutionen systematisch zu entwickeln. Sie bleiben ihren Traditionen unkritisch verhaftet, und sie leben weiterhin in einer Welt der «hybriden» Wesen. Deshalb begründet die erste kategoriale und transzendentale Unterscheidung von Natur und Kultur auch zugleich eine zweite Dichotomie, nämlich die Unterscheidung zwischen Modernen und Vormodernen. Aus moderner Warte erscheinen letztere als Verlierer der Geschichte.

Doch Latours kritische Deutung dreht den Spieß der Geschichte um: Die Modernen sind nämlich kaum mehr in der Lage und erst recht nicht willens, die Hybridität der Existenzen anzuerkennen. Dies habe zur Folge, dass sich die Hybriden gleichsam hinter dem Rücken der Modernen maßlos vermehren und sich damit der demokratischen Kontrolle entziehen. Zwar bekunden die Modernen zunehmend Mühe, in ihren eigenen Handlungsfolgen Natur und Kultur sauber zu trennen, wie dies beispielsweise bei den meisten globalen Umweltproblemen, bei der Gentechnologie

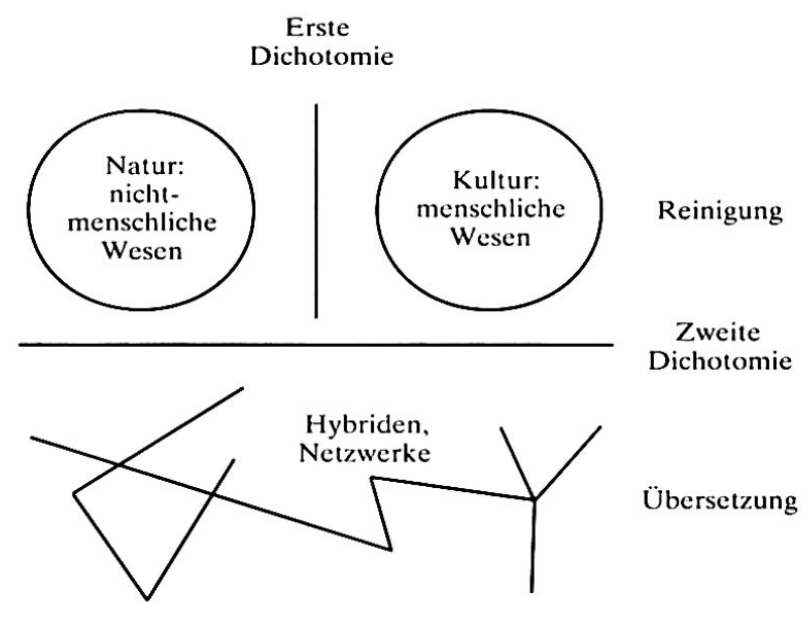

Abb. 2: «Reinigungs- und Übersetzungsarbeit» (nach LATOUR 1995: 20)

The work of purification and translation (according to LATOUR 1995: 20)

Le travail de la purification et de la traduction (d'après LATOUR 1995: 20) oder bei der Beurteilung von Landschaften exemplarisch zu beobachten ist. Doch die Konsequenzen, die daraus gezogen werden, zielen meistens nur daraufhin, die kategoriale Unterscheidung zwischen Natur und Kultur zu verfeinern. Insofern sind die Modernen daran, das Opfer ihrer eigenen Geschichtsschreibung zu werden.

Latour geht es nicht darum, die Segnungen moderner Technologie und des modernen Wohlfahrtsstaates herabzuwürdigen oder rückgängig zu machen. Vielmehr sollen Natur und Kultur als Ergebnisse von Erkenntnispraktiken verstanden werden. LaTOuR spricht von Übersetzungs- (bzw. synonym: Vermittlungs-) und Reinigungspraktiken. Ihr Zusammenspiel lässt sich exemplarisch anhand des wissenschaftlichen Labors illustrieren. Eine Experimentieranordnung vermittelt die unterschiedlichsten Entitäten, wie z.B. Theorien, Wissenschafter, schiefe Ebenen, rollende Kugeln und Messdimensionen. Systematische Variationen der Versuchsanordnungen sollen es erlauben, innerhalb dieser hybriden Settings einen Einfluss der physikalischen Gesetze (Natur) von einem Einfluss der menschlichen Eingriffe (Kultur) zu unterscheiden. Mit der Zeit treten durch die Vermittlungspraktiken im Labor relativ reine Konzeptionen von Gravitation, Masse, Reibung (Natur) einerseits, sowie von mechanischen Technologien (Kultur) andererseits hervor. Weitere Vermittlungsprozesse können die Vorstellungen von Natur und Kultur ebensogut stabilisieren wie revidieren (vgl. Latour 1995: 22-67).

Jedenfalls wäre es aufgrund dieser Sichtweise nicht ratsam, von einer klaren, eindeutigen, fixen Zuordnung von Entitäten zu Natur oder Kultur auszugehen. Keine kategorialen Trennungen, keine transzendentalen Apriori. Vielmehr sind Unterscheidungen stets kontingent, präziser: an den Kontext bestimmter Vermittlungspraktiken gebunden.

\section{Sprachpragmatik und relationales Denken}

LATOUR setzt kulturkritisch an, denn er zeigt auf, inwiefern die Moderne nicht imstande ist, ihren eigenen Anspruch einzulösen. Die durch diese Kritik konstituierte a-moderne Position lässt sich jedoch auch erkenntnistheoretisch einholen. Dies führt zu einer sprachpragmatischen Perspektive, innerhalb derer sich eine Konzeption relationalen Denkens formulieren lässt. Den Schlüssel dazu bildet eine verallgemeinerte Interpretation der Unterscheidung von Reinigungsund Vermittlungspraktiken.

Reinigung lässt sich als Differenzierung, als Unterscheidung im Allgemeinen verstehen. Immer wenn eine Entität bestimmt wird, ist dies ein Ergebnis einer 
Reinigung, bzw. Differenzierung. Bestimmte Aspekte der Welt wurden als Einheit von einer Umwelt unterschieden. Ein derartiger Zugang wird in seltener Reinheit von der Theorie autopoietischer Systeme formuliert: Ein System konstituiert sich, indem es sich von seiner Umwelt unterscheidet (vgl. Maturana \& Varela 1987, Luhmann 1987). Die Beobachtung einer System/Umwelt-Differenz ist die Grundoperation jedes autopoietischen Systems, das Sinn verarbeitet. Durch weitere Beobachtungen lassen sich innerhalb und außerhalb des Systems Differenzen einführen, wobei die Beobachtungen immer als Operationen innerhalb des Systems vollzogen werden.

So wie bei Latour die Reinigungspraktiken stets auf Vermittlungspraktiken beruhen, setzt in der Systemtheorie jede Differenzierung Beobachtung voraus. Beobachtungen sind die Bedingung der Möglichkeit, Unterscheidungen zu treffen. Beobachtungen benötigen aber immer einen Code, d.h. eine Merkmalsdimension, welche die Möglichkeit bestimmter Unterscheidungen vorgibt. Ferner sind Beobachtungen auf «Programme» angewiesen, welche die Anwendung des Codes kontrollieren. Und schließlich lässt sich nur beobachten, was zum Beobachter in einer existenziellen Beziehung steht. Die Theorie autopoietischer Systeme spricht hier von struktureller Koppelung zwischen System und Umwelt. Die Art dieser Beziehung stellt zugleich die Möglichkeiten der Beobachtung dar. Entitäten aus anderen "Welten», wie z.B. Götter und Engel, sind nicht empirisch beobachtbar.

Während sich eine Differenzierung immer eindeutig benennen lässt, lassen sich die hierfür notwendigen Voraussetzungen nicht abschließend angeben. Im Prinzip handelt es sich immer um die Struktur eines unabgeschlossenen Netzwerkes von Beziehungen, also um den Zustand einer gesamten Welt. Allerdings ist es normalerweise hinreichend, einige wenige Beziehungen des Netzwerkes bzw. Aspekte dieses Zustandes zu nennen. Mit ScHüTZ (1982) können wir die Grenzziehungen der Thematisierung als eine Frage verschiedener Relevanzen einer Handlungssituation begreifen. Sprachliche Differenzierungen, also der Sinn von Aussagen, werden nicht etwa durch eine Semantik festgelegt, sondern durch den Kontext der Verwendung letzterer. Die «langue» ist nur das Instrument, das zur Realisation von «parole» benutzt wird. Aufgrund der Kontextrelativität kann nicht auf das Verständnis von Texten vertraut werden. Als sprachpragmatisch lassen sich solche Perspektiven bezeichnen, weil sie die Sprache und die Konstitution von Sinn von der Tätigkeit des Kommunizierens aus thematisieren.

In unserem Zusammenhang ist entscheidend, dass Erkenntnistheorien dieses Zuschnitts ohne transzen- dentale Kategorien operieren. Jede Unterscheidung, jede Bestimmung einer Entität kann als Ergebnis von Praktiken thematisiert werden, muss es jedoch nicht. Dem Denken wird somit kein Fixpunkt, keine Absolutheit welcher Art auch immer, vorausgesetzt. Alles ist relativ. Doch werden zugleich die vielfältigen Voraussetzungen und Folgen des Denkens und Kommunizierens als thematisierbare Umstände mitberücksichtigt. Deshalb ist die Relativität des Differenzierens für das kommunizierende Subjekt keinesfalls eine vollkommene Beliebigkeit: Vielmehr stehen in einer bestimmten Situation immer nur verschiedene Varianten mit ihren spezifischen Konsequenzen zur Wahl.

Die Konzeption kontextbezogener Identitäten hat in den letzten Jahrzehnten beispielsweise in der ökozentrischen Philosophie (vgl. NAESs 1989, Fox 1990, Mathews 1991, Dingler 1994) Aufmerksamkeit erhalten. Identitäten werden dort gemeinhin als «Knoten» im Netzwerk der existenziellen bzw. ökologischen Beziehungen begriffen. Mit derartigen «relationalen» Argumentationen soll der atomistischen Interpretation von Entitäten entgegengetreten werden. Auf diese Weise hofft man, eine moralische Anerkennung nichtmenschlicher Kreaturen begründen zu können.

Mit der Bezeichnung «relational» wird in der ökozentrischen Literatur in der Regel auf «interne Relationen» Bezug genommen. Eine interne Relation besteht zwischen einem $A$ und einem $B$, wenn sich diese nicht unabhängig voneinander definieren lassen. Was oder wie $A$ ist, wird mindestens teilweise durch $B$ bestimmt und umgekehrt. Das heißt, $A$ und $B$ treten in dieser Beziehung nicht als absolut abgrenzbare Einheiten auf. Externe Relationen lassen sich auf verschiedene Weise interpretieren, z.B. als Implikation («Mutter» impliziert «Kind»), als Rückbezüglichkeit (Variablen im Regelkreis), als Unschärferelation (zwischen Beobachtendem und Beobachtetem), als Voraussetzung oder Folge (keine Wirkung ohne Ursache und umgekehrt), oder auch als Koexistenz bzw. Koevolution (Räuber und Beute). Viele ökologische, evolutionäre, sozialisationstheoretische und entwicklungspsychologische Betrachtungsweisen operieren mit internen Relationen.

Externe Relationen beschreiben hingegen ein A vollkommen unabhängig von einem $\mathrm{B}$. Es sind reine Bezeichnungen, wie wir sie üblicherweise von Eigennamen kennen: Anna verweist in keiner Weise auf Berta usw. Immer wenn wir von bestimmten Einheiten oder Entitäten sprechen, operieren wir mit externen Relationen. Eindeutige Differenzen sind immer externe Relationen. Auch die klassische (Aristotelische) Logik beruht auf externen Relationen. Das A muss mit sich selbst identisch sein, und es muss eindeutig vom Nicht-A unterschieden werden können. 
Darüber hinaus darf es kein Drittes neben A und Nicht-A geben (vgl. ReICHERT 1997: 149ff.).

Die Systemtheorie erinnert uns jedoch daran, dass jeder Unterscheidung ein Code, also mindestens eine Merkmalsdimension, im äußersten Fall eine komplexe Semantik, zugrundeliegt. Der Dimension verdanken wir die Möglichkeit, eine Unterscheidung treffen zu können. In der Dimension sind das A und das B als mögliche Ausprägungen schon enthalten; auf der Ebene der Dimension verweisen sie aufeinander, lassen sie sich noch nicht als unabhängige Entitäten betrachten. Dimensionen repräsentieren zunächst interne Relationen.

Insofern als Dimensionen die Bedingungen der Möglichkeit für Differenzierungen darstellen, lassen sie sich als die Ebene der Vermittlungen begreifen. Im wissenschaftlichen Labor müssen beispielsweise Dimensionen von «Kausalität» hergestellt werden, damit sich die kulturelle (das heisst kontrollierte!) Variation der Ursachen mit der Variation der Folgen korrelieren lässt. Dies eröffnet die Möglichkeit, einzelne Ursachen und einzelne Wirkungen als Ausdruck eines gesetzmäßigen, natürlichen Kausalzusammenhanges zu deuten.

Zusammengefasst ergibt sich folgende «Gleichung»:

$\frac{\mathrm{A} \mid \mathrm{B}}{\mathrm{A}<-\mathrm{B}}=\frac{\text { Reinigung }}{\text { Vermittlung }}=\frac{\text { Differenz }}{\text { Dimension }}$

Wir können diesen Bruch durchaus im Sinne von Zähler und Nenner lesen, denn Zählen ist eine abstrakte Form der Differenzierung, bei der die Dimension zwar namenlose, aber gleichwertige Einheiten vorgibt. Für einen Beobachter kann es zweckmäßig sein, sich nur auf den Zähler zu beschränken, und die Dinge so zu betrachten als bestünden sie für sich selbst. Er oder sie operiert dann im Modus der externen Relationen. In vielen Fällen mag es jedoch sinnvoll sein, den ganzen Bruch und die Dinge in ihrem semantischen oder existenziellen Beziehungsgefüge zu berücksichtigen. Dann geraten die Bedingungen der Möglichkeit der Differenzierung bzw. die Vermittlungspraktiken in den Blick. Auf dieser Ebene sind die internen Relationen, die Hybriden, angesiedelt.

Nur die Relevanzen der Handlungssituation entscheiden darüber, in welchem Modus kommuniziert wird. Da jedoch Dimensionen selbst nur als solche erkennbar sind, indem sie von anderen Dimensionen unterschieden werden, können letztlich weder interne noch externe Relationen beanspruchen, eine vollwertige Sicht der Welt abzugeben. Sie bedingen einander, und nur ihre Komplementarität soll die «allgemeine Form relationalen Denkens» heißen.

Indem jede Identität, jeder Begriff durch die Artikulation einer Selektion von semantischen oder existenziellen Beziehungen gebildet wird, lässt sich ihr Sinn als Resultat einer «Fokussierung» aufgrund der Relevanzen eines Handlungskontextes betrachten. Handlungskontext und Relevanzen sind folglich als Ressourcen und Hindernisse der Verständigung zu berücksichtigen. Kommunizierende haben somit im Prinzip immer die Möglichkeit, weitere, andere Beziehungen im Netzwerk der Existenz zu artikulieren. Sie müssen nicht nur, sondern können auch viele Beziehungen unartikuliert lassen.

\section{Normative Konsequenzen}

Da die «allgemeine Form relationalen Denkens» immer nach den Voraussetzungen und dem Zustandekommen von Unterscheidungen fragt, sind ihr alle Kategorien der Kommunikation fragwürdig. Sie setzt keinen «Fixpunkt» des Denkens, keine irgendwie geartete Absolutheit voraus. In diesem spezifischen Sinn (vgl. Habermas 1992: 35ff.) sind Sprachpragmatik und relationales Denken «nachmetaphysisch».

Für normative Verständigungsbemühungen (und für die kritische Sozialwissenschaft) bieten sich folglich keine Anhaltspunkte dafür an, von einer universellen Grundlage der Moral ausgehen zu können. Einvernehmen lässt sich nicht ableiten, sondern es muss aus den unterschiedlichen existenziellen Situationen der involvierten Individuen heraus argumentativ aufgebaut werden. Andere «Anhaltspunkte», andere «Sicherheiten» stehen nicht zur Verfügung. Deshalb kann sich die sprachpragmatische Philosophie auch nicht mehr auf die Entwicklung von Ethiken konzentrieren, sondern betrachtet die ethische Reflexion der Vermittlung widerstreitender ethischer Positionen als ihre eigentliche Aufgabe. Dies führt sie zu prozeduralen Metaethiken, wie der Diskurs- und der Verantwortungsethik (vgl. Apel 1990, Habermas 1983 und 1991, Zierhofer 1994).

Aus dem Umstand, sich nicht einfach auf Elemente einer universellen Moral berufen zu können, darf jedoch nicht abgeleitet werden, es könne keine global akzeptierten Normen geben! Nein, dafür stehen die Chancen gar nicht so schlecht. Das Problem ist nur, dass gemeinsame Vorstellungen von Menschenrechten, Freiheit, Gerechtigkeit, Würde etc. von den Handelnden nicht einfach vorausgesetzt werden können, sondern immer wieder argumentativ erarbeitet werden müssen. Doch genau dadurch lässt sich deren konkrete Auslegung auch wandelnden Umständen anpassen. 


\section{Konsequenzen für den Gesellschaftsbegriff}

Für das moderne Denken lässt sich zwar kein einheitliches Verständnis von «Gesellschaft» reklamieren (vgl. Ritsert 1988), doch verstehen die Sozialwissenschaften des 20. Jahrhunderts «Gesellschaft» meistens im Sinn eines Containers, der alle menschlichen Beziehungen oder Aktivitäten in einer geordneten Weise umfasst. Anhand der dominierenden Strukturen dieser Ordnungen wird versucht, Gesellschaften als Typen zu fassen und zu klassieren. Sofern sich diese Ordnungen als Ergebnis eines «urwüchsigen» Interaktionszusammenhanges deuten lassen, können sie als Kultur eines Volkes begriffen und territorial eingegrenzt werden. Vor allem mit Blick auf «entwickeltere» Gesellschaften, kommt es zu einer Parallelisierung von Gesellschaft und territorial definiertem «Nationalstaat». Die Ordnungen des Zusammenlebens ergeben sich dann durch Formen der Selbstkonstitution; sie beruhen auf bewußten und verhandelbaren Satzungen. Insgesamt laufen jedoch die angedeuteten Versuche, «Gesellschaft» zu fassen, immer wieder auf Abgrenzungsprobleme auf. Sie schließen zuviel oder zuwenig ein, wie Luhmann (1997: 16-35) moniert. Seine Alternative lautet deshalb, Gesellschaft als die Menge aller anschlussfähigen Kommunikationen zu betrachten. In der Tat wird hiermit ein sehr trennscharfes Kriterium für die Unterscheidung von Gesellschaft und Umwelt vorgelegt. Mit LuhmanNs Konzeption dürfte die «Reinigung» des modernen Gesellschaftsbegriffes ihren vorläufig letzten Stand erreicht haben.

Der Ausschluss aller nicht-menschlicher Seiten der Existenz aus dem modernen Gesellschaftsbegriff erfolgt durchwegs ohne explizite Begründung. Das heißt, die Unterscheidung Gesellschaft/Umwelt wird mit der transzendentalen Dichotomie von Kultur/Natur parallelisiert. Exemplarisch manifestiert sich dieses Verständnis in der umweltethischen Debatte (vgl. von der Pfordten 1996, Zimmermann et al. 1993). Denn nur der Umstand, dass Gesellschaft mit großer Selbstverständlichkeit als rein menschliche Sphäre verstanden wird, kann erklären, weshalb sich nicht anthropozentrische ethische Positionen, sondern nur ihre patho-, bio- und ökozentrischen Alternativen um ihre Begründung bemühen müssen. Man kann die umweltethische Debatte daher über weite Strecken so verstehen, dass in ihr die moralische Relevanz via die Frage der Zugehörigkeit zu Gesellschaft verhandelt wird.

Aus a-moderner Perspektive ist zunächst jeder Ausschluss begründungspflichtig, dies um so mehr, als er gegenüber dem Einschluss eine anspruchsvollere Position darstellt. Wie lässt es sich rechtfertigen, dass Tiere, Pflanzen, Maschinen, sonstige Artefakte, aber auch alle anderen «natürlichen» Entitäten und Prozesse nicht als Mitglieder der Gesellschaft betrachtet werden? Wie lässt sich die Haltung vertreten, sie nicht als moralisch relevant zu erachten? Welche Zwecke werden wohl mit der anthropozentrischen Fokussierung von Gesellschaft und Moral verfolgt?

Aus relationaler Sicht lässt sich nichts a priori aus der Gesellschaft ausschließen. Konsequenterweise wird sie auch nicht mehr a priori als Menge verstanden. Zwar wird nicht ausgeschloßen, dass eine Konzeption von Gesellschaft als «Behälter» für bestimmte Zwecke adäquat sein könnte. Gesellschaft als Grundbegriff für die Sozialwissenschaften im Allgemeinen bezieht sich hingegen sinnvollerweise auf diejenigen Strukturen der Koexistenz, die einem Beobachter als besonders relevant erscheinen. Auch wenn aufgrund des Erkenntnisinteresses primär die Formen des Zusammenlebens der Menschen untereinander ins Auge gefasst werden, ist es problematisch, Strukturen, die sich auf Interaktionen zwischen Menschen und anderen Entitäten beziehen, aus dem Gesellschaftsbegriff von vornherein ausschließen zu wollen. Dieser Sicht entsprechen auch gängige Gesellschafts-Typologien: Wenn von Jägern und Sammlerinnen, von Viehzüchtern, von Nomaden, von Garten- und Ackerbauern, aber auch von Industrie- und High-Tech-Gesellschaften die Rede ist, dann werden Formen der Interaktion mit nicht-menschlichen Entitäten zur Klassierung bemüht. Was die moderne Gesellschaftstheorie vor die Türe setzt, führt ihre empirische Sozialforschung durch den Dienstboteneingang wieder ein.

Welche existenziellen Beziehungen jeweils als Gesellschaft thematisiert werden und welche nicht, ist eine Frage der Relevanzen der Beobachter und ihres Kommunikationszusammenhanges. Weil sich immer auch andere Beziehungsgefüge artikulieren lassen, macht es kaum Sinn, nach einer allgemeingültigen Charakterisierung einer Gesellschaft zu fragen. Die Konzeption der Fokussierung impliziert somit eine Pluralität von Zugängen. Verhandelbar ist daher nicht nur, welche Gültigkeit bestimmte Beschreibungen beanspruchen können, sondern auch, für welche Probleme sie als hinreichende Instrumente zu betrachten sind und für welche nicht. In diesem Sinn kann die Vielfalt gegenwärtig kursierender gesellschaftlicher Selbstbeschreibungen (Risikogesellschaft, Informationsgesellschaft, Freizeitgesellschaft usw.) ebenso als Widerspruch oder als Konkurrenz von Deutungen, wie als komplementäre Fokussierungen verstanden werden.

Abgrenzungen von Gesellschaft sind nur unter der Bedingung möglich, dass sich die artikulierte Struktur selbst auf eine Klasse von Entitäten, z.B. auf Individuen (Verwandtschaft) oder Tätigkeiten (Kommunikation) bezieht. Und nur sofern diese Entitäten eine körperliche Existenz aufweisen - was keine Notwen- 
digkeit ist - besteht die Chance, Gesellschaften auch (physisch-)zeit-räumlich unterscheiden zu können.

Erst die Teilhabe an einer Existenz- und Kommunikationsgemeinschaft versetzt potenzielle Beobachter in die Lage, semantische und existenzielle Beziehungen zu artikulieren. Auf diese Weise lässt sich der Gedanke, dass nur der sozialisierte Beobachter auch die Fähigkeit besitzen kann, das Soziale diskursiv zu durchdringen, sprachpragmatisch und relational fassen. Oder mit LuhmanN: Es gibt keinen Ort außerhalb der Gesellschaft, von der aus sie sich als Ganzes beobachten ließe (vgl. 1997: 88). Wie wir an der Koexistenz teilhaben, welche Vermittlungen wir eingehen, bestimmt letztlich unsere Möglichkeiten, die Koexistenz zu artikulieren.

\section{Konsequenzen für die akademische Ordnung und die Position der Geographie}

Indem Natur und Kultur nicht mehr im modernen Sinn als transzendentale Dichotomie verstanden werden, wird auch die daran gekoppelte akademische Arbeitsteilung fragwürdig. Zumindest lässt sich aus a-moderner Perspektive eine Trennung zwischen Naturwissenschaften auf der einen Seite sowie Geistes- und Sozialwissenschaften auf der anderen Seite nicht mehr durch Verweis auf den Erkenntnisaktivitäten vorausliegende Forschungsgegenstände begründen. Denn die Gegenstände der Forschung werden aus a-moderner Sicht als Entitäten betrachtet, die durch das Forschungsinteresse und die Forschungspraxis erst als solche bzw. als Realität konstituiert werden.

Vor allem die Umweltprobleme, aber auch die Biound Informationstechnologien führen zu Forschungsgegenständen, in denen sich Natur und Kultur vermengen. Nachdem sich die Probleme nicht angemessen bearbeiten lassen, indem man sie vollumfänglich entweder der einen oder der anderen Seinssphäre zurechnet, wird in der Moderne der Ruf zuerst nach inter- und später nach transdisziplinärem Arbeiten laut. Zwar gilt Transdisziplinarität gegenwärtig als chic, doch drückt sich darin eigentlich nichts anderes aus als die Unangemessenheit der bestehenden akademischen Ordnung. Wozu organisiert sich die Wissenschaft noch in Form von Disziplinen, wenn sie anschließend überwunden werden sollen? So besehen, ist der Ruf nach disziplinenübergreifender Kooperation eine Bankrotterklärung des Systems wissenschaftlicher Arbeitsteilung. Darüber hinaus wird sich erst noch weisen müssen, ob transdisziplinäre Forschung nicht wieder gerade diejenigen konstitutiven Bedingungen der Moderne fortschreibt, welche die Existenz komplexer Hybriden erst zum Problem machen. Andernfalls wäre Transdisziplinarität selbst als Teil des
Problems zu verstehen, gegen das sie ins Leben gerufen wurde.

Aus a-moderner Perspektive stellen Disziplinen keine fixen Grenzen dar. Sie lassen sich deshalb auch nicht a priori (hier vor allem: unabhängig vom einzelnen Fall) als Hindernisse deuten. Vielmehr kann in ihnen auch ein historisch gewachsenes Kapital - nämlich eine kontinuierlich entwickelte Kompetenz des Differenzierens - gesehen werden. Dieses Kapital kann dazu dienen, bestimmte und daher zugleich unterschiedliche Gegenstandsbereiche sichtbar und verfügbar zu machen. Folglich kann es weniger darum gehen, neuartige Probleme mit grenzüberschreitender Kooperation anzupacken, denn genau dann würden die Grenzen ja dysfunktional bzw. unzweckmäßig, sondern mehr darum, den Verlauf der Grenzen und ihre Permeabilität produktiv zu wandeln. Disziplingrenzen könnten sich auch laufend denjenigen Gegenständen anpassen, deren Konturen gerade eben erst sichtbar werden. Mit anderen Worten: Disziplinen ließen sich als die Form begreifen, mit Hilfe derer sich Beobachtungsfähigkeit durch Selektivität weiterentwickeln lässt.

Im Übrigen gilt es zu beachten, dass sich die fachlichen Probleme in der Regel als Inkompatibilität von «Theorien» darstellen. Doch terminologische und konzeptuelle Anschlussschwierigkeiten halten sich keineswegs an Disziplingrenzen: So «verstehen» sich Physik und Chemie bestens, während sich verschiedene Soziologien konkurrenzieren, ja «bekämpfen». Verständigungsprobleme zwischen verschiedenen wissenschaftlichen Perspektiven beruhen oftmals nicht auf Disziplingrenzen, sondern auf unterschiedlichen Terminologien und Wissenschaftsverständnissen, die quer zur akademischen Arbeitsteilung verlaufen. Für solche Schwierigkeiten stellt Inter- oder Transdisziplinarität selbst im modernen Denkrahmen keine vernünftige Lösung dar. Vielmehr sind dafür Kritik- und Vermittlungsstrategien zwischen den Ansätzen gefragt, doch schnelle Antworten sind auf diese Weise nicht zu erwarten.

Wird die Konzeption, Disziplinen konstituierten sich durch einen vor ihren Augen existierenden Gegenstand und das damit implizierte Zuschauer-Modell der Erkenntnis (RoRTY 1992: 39) zugunsten einer sprachpragmatischen Perspektive aufgegeben, dann konstituieren Disziplinen aufgrund ihrer eigenen Relevanzen zugleich sich selbst und ihre eigenen Forschungsgegenstände. Wird darüber hinaus, und konsequenterweise, auch die transzendentale Dichotomie von Natur und Kultur verworfen, dann müssen sich der Thematisierung von Gegenständen, die zugleich Natur und Kultur sind, weder prinzipielle noch institutionelle Hindernisse entgegenstellen. Selbstverständlich sind 
Denkgewohnheiten und Terminologien immer noch in mühsamer Kleinarbeit weiterzuentwickeln. Aber die akademische Organisationsform «Disziplin» muss dafür kein Hindernis sein, sondern kann als Instrument hierfür genutzt werden.

Gerade darin könnte nun eine Chance für die Geographie liegen. Denn diese Disziplin beschäftigt sich traditionell mit Phänomenen und Problemen, die an den «Kontaktflächen» von Natur und Kultur angesiedelt sind, sei es als Verhältnis von Mensch und Raum, als (Kultur-)Landschaften, als Sphäre zwischen Sinn und Materie (vgl. Gren 1994) oder als Zusammenspiel von physischer, subjektiver und sozialer Welt (vgl. WERLEN 1987).

Aus moderner Warte wurde die Geographie bis hin zu Raumwissenschaft und verhaltenstheoretischem Ansatz durch ein vormodernes Denken dominiert, denn sie folgte keiner strikten Unterscheidung von Natur und Kultur im Sinne autonomer Seinssphären. Folglich war sie auch nicht im Stande, sich intern nach den methodologischen Maßstäben der Natur- oder Sozialwissenschaften zu differenzieren, sondern organisierte sich anachronistisch als einheitliche Disziplin. WerLens (1987) Grundlegung der Sozialgeographie durch Rückgriff auf eine kategoriale Trennung von physischen Sachverhalten, subjektivem- und sozialem Sinn, sowie seine (1995) Parallelisierung von traditionellen vs. modernen Lebensformen mit entsprechenden geographischen Ansätzen repräsentiert die moderne Kritik systematisch und konsequent.

Während die «vormoderne» Einheits-Geographie dazu tendiert, Kultur auf Natur zu reduzieren, und dadurch ständig Gefahr läuft, Formen des Umweltdeterminismus oder des normativen Naturalismus zu verfallen, spaltet sich die moderne Geographie in eine physische und eine soziale. Dies geschieht allerdings primär dadurch, dass sich die Humangeographie zunehmend als Sozialwissenschaft versteht. Wissenschaftlichkeit ist der Gewinn, Schisma und Identitätskrise sind der Preis, den die Geographie für ihre Modernisierung zu bezahlen hat. Denn wenn sich physische Geographie und Humangeographie mit verschiedenen Gegenständen unter Anwendung verschiedener Forschungsmethoden befassen, lässt sich die Legitimität der Einheit der Disziplin im Rahmen modernen Denkens kaum mehr begründen. Leider artikuliert sich das Problem der institutionellen Zuordnung der Disziplin zu einer natur- oder sozialwissenschaftlichen Fakultät nicht nur als Streit um das Selbstverständnis innerhalb der Disziplin, sondern gelegentlich auch als unangemessene Kritik an Diplomarbeiten oder Dissertationen aus dem jeweils «anderen» Bereich der Fakultät. Man könnte deshalb auch sagen, im Rahmen der modernen akademischen Arbeitsteilung wurde eine spezifische Form der Ignoranz institutionalisiert - eben der blinde Fleck der Modernen.

Im Rahmen a-modernen Denkens bieten sich der Geographie neue Möglichkeiten an, sich als fachliche Einheit zu profilieren. Sie könnte gerade diejenigen Probleme der Moderne bearbeiten, die sich nach moderner Interpretation an den Grenzen von Natur und Kultur ansiedeln, also Probleme, die sich beispielsweise auf den Metabolismus der «Gesellschaft», auf ihre Infrastruktur, ihre Ressourcennutzungen oder ihre zeit-räumlichen Organisationsweisen («alltägliche Regionalisierungen») beziehen. Lösten sie sich von der Auffassung, nur ein exklusiv beanspruchbarer Forschungsgegenstand verbürge ihre Existenz, so müssten weder die physische Geographie noch die Humangeographie befürchten, sich in anderen natur- oder sozialwissenschaftlichen Disziplinen aufzulösen. Vielmehr könnten sie sich als Fokussierungen innerhalb der Geographie begreifen und ihre Berechtigung im erfolgreichen Absatz von Dienstleistungen in Forschung und Bildung sehen. Die nützliche Artikulation von Beziehungen im Netzwerk der Existenz würde die selbstgewählte Disziplinierung legitimieren. Hierfür müssten allerdings auch entsprechende Kompetenzen aufgebaut werden.

Mit Entschiedenheit bleibt jedoch festzuhalten, dass die methodologische Unterscheidung von «Natur-» und «Sozialwissenschaften», also das, was GidDENS (z.B. 1984: 95) die doppelte Hermeneutik nennt, keineswegs außer Kraft gesetzt wird. Vereinfacht gesprochen geht es darum, dass es ebenso sinnlos ist, mit Landschaften Interviews zu führen, wie Gedanken im Luftbild lesen zu wollen. In Hinblick auf die Erklärung von Prozessen und Aktivitäten, auf die daraus abgeleitete Erhebung von Daten und auf deren Interpretation ist die spezifische Handlungsfähigkeit oder «agency» der beobachteten Entitäten zu berücksichtigen: Wird die Entität als unbelebter Gegenstand oder Prozess, als Organismus, als Organismus mit Bewusstsein oder als sprachfähiges Wesen betrachtet? Man könnte in Anlehnung an GidDENs vielleicht von einer «differenzierten Hermeneutik» sprechen.

Die Geographie kann sich komplexe hybride Forschungsgegenstände zurechtlegen und sich damit im Rahmen der akademischen Arbeitsteilung als Einheit behaupten. Doch sie wird in der empirischen Forschung nicht darum herum kommen, den Forschungsgegenständen entsprechend, verschiedene Methodologien kompetent zu handhaben. In diesem Sinn kann eine a-modern verstandene Geographie nicht auf die methodologischen Vorarbeiten der modernen Natur-, Geistes- und Sozialwissenschaften verzichten. 


\section{Literatur}

APEL, K. (1990): Diskurs und Verantwortung. - Frankfurt am Main: Suhrkamp.

BECK, U. (1991): Der Konflikt der zwei Modernen. - In: ders.: Politik in der Risikogesellschaft. - Frankfurt am Main: Suhrkamp: 180-195.

DINGLER, J. (1994): Deep Ecology - Utopie oder ein neuer Weg zur Lösung der Umweltkrise. Analyse eines ökozentrischen Beitrags zur Umweltdiskussion. - Magisterarbeit, Universität Konstanz, Fakultät für Politik und Verwaltungswissenschaft.

Fox, W. (1990): Toward a Transpersonal Ecology. Developing New Foundations for Environmentalism. - Foxhole, Dartington: Green Books.

Giddens, A. (1984): Interpretative Soziologie. Eine kritische Einführung. - Frankfurt am Main: Campus.

GrEN, M. (1994): Earth writing. Exploring representation and social geography in-between meaning/matter. - Department of Human and Economic Geography, University of Gothenburg.

Habermas, J. (1983): Diskursethik - Notizen zu einem Begründungsprogramm. - In: Ders.: Moralbewusstsein und kommunikatives Handeln. - Frankfurt am Main: Suhrkamp: 53-125.

Habermas, J. (1990): Die Moderne - ein unvollendetes Projekt. - In: ders.: Die Moderne - ein unvollendetes Projekt. Philosophisch-politische Aufsätze 1977-1990. - Leipzig: Reclam: 32-54.

Habermas, J. (1991): Erläuterungen zur Diskursethik. - Frankfurt am Main: Suhrkamp.

Habermas, J. (1992): Nachmetaphysisches Denken. Philosophische Aufsätze. - Frankfurt am Main: Suhrkamp.

HaraWAY, D. J.(1995): Monströse Versprechen. CoyoteGeschichten zu Feminismus und Technowissenschaft. - Hamburg: Argument.

Latour, B. (1995): Wir sind nie modern gewesen. Versuch einer symmetrischen Anthropologie. - Berlin: Akademie.

Law, J. \& J. Hassard (eds) 1999: Actor Network Theory and after. - Oxford: Blackwell.

LuhmanN, N. (1987): Soziale Systeme. Grundriss einer allgemeinen Theorie. - Frankfurt a. Main: Suhrkamp. Luhmann, N. (1997): Die Gesellschaft der Gesellschaft. 2 Bände. - Frankfurt am Main: Suhrkamp.

Mathews, F. (1991): The Ecological Self. - London: Routledge.

Maturana, H. R. \& F. J. Varela (1987): Der Baum der Erkenntnis. - Bern: Scherz.

NAESS, A.; translatend and revised by Rothenberg, D. (1989): Ecology, community and lifestyle. Outline of an ecosophy. - Cambridge, Mass.: Cambridge University Press.

Pfordten, D. von der (1996): Ökologische Ethik. Zur Rechtfertigung menschlichen Verhaltens gegenüber der Natur. - Reinbek bei Hamburg: rororo.

Reichert, D. (1997): Zur Vorgeschichte einer Geogra- phie von Menschen. Zu Selbstreferenz ohne Zirkularität und in sich geschlossene Einheit. - Dissertation, Universität Wien.

RITSERT, J. (1988): Gesellschaft. Einführung in den Grundbegriff der Soziologie. - Frankfurt am Main: Campus.

RoRTY, R. (1992): Introduction. Metaphilosophical Difficulties of Linguistic Philosophy. - In: RoRTY, R. (ed.): The Linguistic Turn. Essays in philosophical Method.Chicago: University of Chicago Press: 1-39.

Schürz, A. (1982): Das Problem der Relevanz. - Frankfurt am Main: Suhrkamp.

WeLSCH, W. (1997) (1986): Unsere postmoderne Moderne. - Berlin: Akademie.

Werlen, B. (1987): Gesellschaft, Handlung und Raum. - Stuttgart: Steiner.

WERLEN, B. (1995): Sozialgeographie alltäglicher Regionalisierungen. Band 1: Zur Ontologie von Gesellschaft und Raum. - Stuttgart: Steiner.

ZIERHOFER, W. (1994): Ist die kommunikative Vernunft der ökologischen Krise gewachsen? Ein Evaluationsversuch. - In: Zierhofer, W. \& D. Steiner (Hrsg.): Vernunft angesichts der Umweltzerstörung. - Opladen: Westdeutscher Verlag: 161-194.

Zierhofer, W. (1999): Geographie der Hybriden. - In: Erdkunde 53/1: 1-13.

Zimmerman, M., Callicott, J. B., Sessions, G., Warren, K. \& J. ClaRK (eds) (1993): Environmental Philosophy. From Animal Rights to Radical Ecology. - Englewood Cliffs, New Jersey: Prentice Hall.

\section{Zusammenfassung: A priori ohne Apriori A-moderne, Sprachpragmatik und Geographie}

A-moderne Denkansätze fragen systematisch nach der Konstitution von Entitäten jeglicher Art durch eine erkennende oder sprechende Instanz. Insofern radikalisieren sie moderne Perspektiven. Indem sie jedoch auch die Dichotomie von Natur und Kultur, die stillschweigend vorausgesetzt die Grundlage für das Selbstverständnis der Moderne bildet, als kontextrelative Konstruktion betrachten, setzen sie sich markant von der Moderne ab. Gerät die Unterscheidung von Natur und Kultur in Fluss, kann sie auch nicht mehr zur Ordnung akademischer Aktivitäten herangezogen werden. Für die Geographie könnte sich daraus die Chance ergeben, sich als eine a-moderne Disziplin zu profilieren, die sich der in der Moderne problematischen Mischphänomene zwischen Natur und Kultur kompetent annimmt.

\section{Summary: A priori without Apriori}

\section{A-modernity, Language Pragmatics and Geography}

A-modern makes use of diagnostic or communicative means to systematically investigate entities of every kind. In this respect they radicalize modern perspectives. Yet, by regarding the dichotomy of nature and 
culture - the tacit foundation of modernity - as a contextual construction, they differ considerably from modern perspectives. As soon as the distinction between nature and culture becomes fluid, it can no longer provide a basic orientation for the organization of academic activities. From this might arise the chance for geography to distinguish itself as an a-modern discipline which is qualified to devote itself to the problematic hybrid phenomena between nature and culture.

\section{Résumé: A priori sans apriori - Non-modernité, pragmatique de la parole et géographie}

Les approches non-modernes cherchent à savoir, d'une manière systématique, comment des entités de toute sorte sont constituées par l'interprétation ou le discours d'un acteur humain ou non humain. C'est ainsi qu'elles rendent encore plus radicaux les principes émancipateurs des modernes. Par contre, en considérant la dichotomie entre Nature et Culture comme une construction contextuelle (dichotomie qui implicitement constitue l'époque moderne), elles tranchent nettement avec la pensée moderne. Au moment où la ligne de séparation entre Nature et Culture commence à perdre de sa netteté, on ne peut plus faire appel à elle pour organiser les activités académiques. C'est là que pourrait se situer une chance pour la géographie à l'avenir: se définir comme discipline nonmoderne et s'occuper d'une manière compétente de tous ces phénomènes hybrides entre Nature et Culture, qui, pour les modernes, posent problème.

Dr. Wolfgang Zierhofer, Department of Human Geography, University of Nijmegen, P.O. Box 9108, NL$6500 \mathrm{HK}$ Nijmegen.

e-mail:W.Zierhofer@bw.kun.nl 